\title{
O. L. VOLOSHENIUK
}

\section{GLOBAL TRENDSIN THE DEVELOPMENT OF LOW-ORBIT SPACE SYSTEMS FOR OPTOELECTRONIC EARTH OBSERVATION}

\author{
Institute of Technical Mechanics \\ of the National Academy of Sciences of U kraine and the State Space Agency of U kraine \\ 15 Leshko-Popel St., Dnipro 49005, U kraine; e-mail: oksana.volosheniuk@ gmail.com
}

\begin{abstract}
The aim of this work is to identify the global trends in the use of low-orbit spacecraft constellations, which have a number of substantial advantages in Earth remote sensing (ERS). Much attention is given to the construction of large constellations of single- and different-type spacecraft by foreign companies and operators, such as Digital Globe, Planet Labs, Black Sky, Satellogic S.A. etc., and to the plans of deployment of constellations of this type in the USA, China, Japan, Canada, Europe, and other countries. The characteristics of the various, mainly commercial, low-orbit constellations of optoelectronic Earth observation spacecraft put into orbit over the past five years are considered. It is shown that low-orbit spacecraft constellations can be used to good advantage in the solution of numerous socio-economic problems, such as geodesy and cartography, urban development, transport infrastructure, crop estimate, environmental monitoring, health monitoring, etc., and new problems aimed at prompt continuous monitoring of various objects. The paper presents a comparative analysis of the technical implementations and ways of achievement of the main target spacecraft characteristics, such as information performance, capture range, revisit time, geodetic connection accuracy, imaging immediacy, and the possibility of stereo and video imaging by satellite constellations. It is shown that the construction of large loworbit constellations is a new trend in the world astronautics, which requires systematic methods for their design and control. The results obtained allows one to make recommendations on the design of low-orbit constellations of home ERS spacecraft, in particular on the development of orbit determination models and algorithms and spacecraft dynamics models.
\end{abstract}

Keymords: Earth remote sensing, low-orbit spacecraft constellation, optoelectronic observation system, spatial resolution, information performance, immediacy.

1. Dvorkin B. A., Dudkin S. A. Newest and prospective Earth remote sensing satellites. Geomatika. 2013. No. 2. Pp. 16-36. (in Russian).

2. Danilova T. D., Nafieva E. N., Tarasova P. D. Results of the ERS spacecraft launches in 2018 and further prospects. Geoprofi. 2019. No. 1. Pp. 16-19. (in Russian).

3. Baklanov A. I. New horizons of space systems for high-resolution optoelectronic Earth observation. Raket.-Kosm. Priborostr. Inf. Sist. 2018. No. 3. Pp. 17-28. (in Russian), https://doi.org/10.30894/issn2409-0239.2018.5.3.17.28

4. Planet Labs Inc. URL: www.planet.com (last accessed on August 20, 2020).

5. RapidEye imagery product specifications. URL: https://www.planet.com/products/satelliteimagery/files/160625-RapidEye\%20Image-Product-Specifications.pdf (last accessed on June 15, 2020).

6. PlanetScope Full Archive. URL: https://earth.esa.int/web/guest/data-access/view-data-product//article/planetscope-full-archive (last accessed on March 15, 2020).

7. Gansvind I. N. Small spacecraft: a new line in space activities. Mezdunar. Nauchno-Issled. Z. 2018. No. 12(78). Pp. 84-91. (in Russian).

8. \$22 Billion Market Value for Small Satellites over Next Ten Years 3,600 smallsats expected to be launched through 2025. URL: https://euroconsult-ec.com/7_July_2016 (last accessed on July 14, 2020).

9. About Digital Globe. URL: https://www.digitalglobe.com/company/about-us (last accessed on April 16, 2020). 
10. WorldView- Legion. URL: https://www.maxar.com/splash/worldview-legion (last accessed on May 12, 2020)

11. WorldView-Scout. URL: https://space.skyrocket.de/doc_sdat/worldview-scout.htm (last accessed on May 12, 2020).

12. BlackSky Global Commercial Imaging Constellation. URL:

https://directory.eoportal.org/web/eoportal/satellite-missions/b/blacksky-constellation (last accessed on May 18, 2020).

13. SpaceView Small Satellite Imaging Solutions. URL: https://www.harris.com/solution/spaceview (last accessed on May 24, 2020).

14. Sat and parts. URL: http://www.charmingglobe.com/EWeb/product2.aspx?tid=33\&id=23 (last accessed on August 19, 2020).

15. Satellite Programme: Gao Fen. URL: https://www.wmo-

sat.info/oscar/satelliteprogrammes/view/240 (last accessed on August 19, 2020).

16. Satellogic. URL: https://en.wikipedia.org/wiki/Satellogic (last accessed on July13, 2020).

17. Ming Li. Remote sensing satellite planning in Chinese National Space infrastructure for next eight years. Proceedings of the 68th International Astronautical Congress (IAC 2017), Australia, 2013. IAC13-B1.2.1.

18. GaoJing / SuperView Earth Observation Constellation. URL:

https://directory.eoportal.org/web/eoportal/satellitemissions/g/gaojing (last accessed on July 21, 2020).

19. GaoJing-1 01, 02, 03, 04 (SuperView 1) URL: https://mapgroup.com.ua/kosmicheskie-apparaty/37kitaj/1642-gaojing-1-01-02-03-04-superview-1 (last accessed on July 22, 2020).

20. Mission Overview GaoFen-5. URL:

http://ceos.org/document_management/Virtual_Constellations/ACC/Meetings/AC-VC-

12/Day\%201/5.\%20Linagfu\%20Chen\%20-\%20Gaofeng-5\%201013.pdf (last accessed on June 21, 2020).

21. Jilin-1 Kuanfu-01 (Jilin-1 Wideband-01). URL: https://space.skyrocket.de/doc_sdat/jilin-1wideband-01.htm (last accessed on July 23, 2020).

22. ASNARO (Advanced Satellite with New System Architecture for Observation). URL:

https://directory.eoportal.org/web/eoportal/satellite-missions/a/asnaro (last accessed on July 25, 2020).

23. DMS 3/TripleSat constellation-1,2,3. URL: https://innoter.com/sputniki/dmc3-triplesat-constellation1-2-3/ (last accessed on July 27, 2020).

24. CSO-1 Earth remote sensing satellite. URL: https://ecoruspace.me/CSO-1.html (last accessed on July 28, 2020). (in Russian).

25. Panin D. N., Karyagina M. V., Malakhova D. S., Salmina Ya. A. Experience of international cooperation in Earth remote sensing: problems and prospects. Mezdunar. Nauchno-Issled. Z. 2020. No. 1(91). Pp. 53-56. (in Russian).

26. Sfera satellite constellation. URL: https://forum.nasaspaceflight.com/index.php?topic $=45849.0$ (last accessed on July 28,2020 ).

27. OptiSAR (Optical and SAR) Commercial Constellation of UrtheCast. URL:

https://directory.eoportal.org/web/eoportal/satellite-missions/o/optisar (last accessed on July 9, 2020). 
